\title{
ENERGY AWARE CLUSTER BASED ROUTING SCHEME FOR WIRELESS SENSOR NETWORK
}

\author{
Sohini ROY ${ }^{1}$
}

\begin{abstract}
Wireless Sensor Network (WSN) has emerged as an important supplement to the modern wireless communication systems due to its wide range of applications. The recent researches are facing the various challenges of the sensor network more gracefully. However, energy efficiency has still remained a matter of concern for the researches. Meeting the countless security needs, timely data delivery and taking a quick action, efficient route selection and multi-path routing etc. can only be achieved at the cost of energy. Hierarchical routing is more useful in this regard. The proposed algorithm Energy Aware Cluster Based Routing Scheme (EACBRS) aims at conserving energy with the help of hierarchical routing by calculating the optimum number of cluster heads for the network, selecting energy-efficient route to the sink and by offering congestion control. Simulation results prove that EACBRS performs better than existing hierarchical routing algorithms like Distributed Energy-Efficient Clustering (DEEC) algorithm for heterogeneous wireless sensor networks and Energy Efficient Heterogeneous Clustered scheme for Wireless Sensor Network (EEHC).
\end{abstract}

Keywords: Wireless sensor network, Energy-Efficient, Routing scheme, Lifetime, Level based, Event driven.

\section{Introduction}

A wireless sensor network is composed of numerous micro electro-mechanical sensor nodes, which are densely deployed in an inaccessible area. The nodes in the sensor network can be static or slightly mobile in nature. The sensors are mainly dedicated to the chores of sensing the environmental events like earthquake, fire, blasts, etc. and sending them to a

${ }^{1}$ Department of Computer Science and Technology, Indian Institute of Engineering Science and Technology (IIEST) shibpur (formerly Bengal Engineering and Science University), Howrah 711103, Email: roysohini266@gmail.com, Phone:+918961145502 
common sink or the base station. Hierarchical routing algorithms like LEACH [2], HEED [9] etc. select a node as a head node from a group of nodes in the network and bestow upon it the task of data aggregation. Data aggregation is an operation performed by the head node or cluster head node in which the data sensed by other nodes in a group or cluster is examined by the head node and they are aggregated, compressed and filtered from redundancy. The aggregated data is then carried to the base station either in a single-hop as in LEACH [2], DEEC [6], and EEHC [4] by the cluster head or in multiple-hops as in CERP [7], MRP [10] etc. Thus, cluster heads play an important role in energy conservation by performing data aggregation as well as sending the aggregated data to the sink. However, selection of required number of cluster heads is a vital requirement in hierarchical routing algorithms. Selection of more than the required number of cluster heads will result in more energy draining of the network. On the other hand, choice of less than the required number of cluster heads will suffer from poor coverage. The proposed scheme EACBRS thus adopts a method of selecting the optimum number of cluster heads for the network. The nodes that are adjacent to the base station are the only communicating links with the base station and thus must be involved in any route if multi-hop routing protocol is followed. This leads to depletion of more energy of the nodes that are closer to the base station. EACBRS thus takes up a level-based approach to deal with this problem keeping the base station at level zero. In this algorithm the nodes of the lowermost level are treated differently to conserve energy. The base station initiates the level formation process and it takes place only once in the lifetime of the network. The level formation is followed by the required number of cluster head selection in each level. Heterogeneity of the nodes is considered while the cluster head selection process. The duty of cluster head is switched between the nodes of a cluster for proper distribution of energy. Cluster formation phase is initiated by the selected cluster heads and is repeated only when a new node enters into the network region.

In this paper, section 2 contains a review on past works, done in the area of energy efficient cluster based routing protocols in wireless sensor network. A detailed description of the proposed protocol is given in section 3 and the case study is done in section 4 . In section 5 the performance of the work is evaluated and compared with existing protocols. Finally section 6 draws the conclusion for the paper.

\section{2. $\quad$ Related Work}

In this section various hierarchical protocols offering energy efficiency in wireless sensor network are discussed.

Low Energy Adaptive Clustering Hierarchy (LEACH) [2] is a homogeneous clustering algorithm. It treats all the nodes without discrimination and forms clusters with only singlehop nodes based on the received signal strength. This kind of cluster formation increases the complexity of routing. In this protocol a number of nodes are randomly selected as cluster heads. The role of cluster head is rotated among the nodes of the cluster to increase the network lifetime. 
In HEED [9] proper energy dissipation is achieved by selecting the cluster heads on the basis of residual energy. Although ignoring the network structure brings about uneven energy consumption throughout the network.

Design of a distributed energy-efficient clustering algorithm for heterogeneous wireless sensor networks (DEEC) [6] proposes an energy efficient clustering algorithm scheme where cluster heads are selected periodically by a probability value. The probability is based on the ratio between the residual energy of each node and the average energy of the network. The authors of this paper have considered a heterogeneous network where nodes are equipped with different amount of energy and have also taken into account the dynamic nature of the network where new nodes are added with more residual energy. The heterogeneity of the network has helped in prolonging the network lifetime and specially the stability period. The algorithm proposed by this paper is also fit for the multilevel heterogeneous environment. However, direct sending of data to the sink by the cluster heads is an infeasible approach for larger networks and this also reduces the energy of the sender considerably.

Energy Efficient Heterogeneous Clustered scheme for Wireless Sensor Network [4] enhances the performance with the introduction of heterogeneity and by the selection of cluster heads based on certain parameters like average distance of a cluster head from its member nodes, average distance of the cluster head from the base station and the transmission range of the amplifier. However, assuming that every cluster head can forward the aggregated data to the base station in a single hop and overlooking the hostility of the working environment of the sensors are the drawbacks of the scheme. EEHC adopts energy heterogeneity by supplying line power to the sensor nodes or replacing their battery. Such actions are impossible for the sensor nodes operating in a remote place. Otherwise, there was no need for energy-aware protocols for sensor network.

Cluster based Event Driven Routing Protocol (CERP) for Wireless Sensor Network [7] proposes an event-driven cluster formation scheme. The cluster heads are selected from the nodes of a cluster on the basis of parameters like residual energy and average distance from the other nodes in the cluster. Multi-hop path using Dijkstra's shortest path finding algorithm is followed in sending the aggregated data to the sink from each cluster head.

A Multipath Routing Protocol Based on Clustering and Ant Colony Optimization for Wireless Sensor Networks [10] is based on Multipath Routing Protocol (MRP). The algorithm follows an event-driven cluster formation scheme and the node placed at the focus of the event is selected as the cluster head node. The total load of the cluster head is divided into smaller parts and multiple routes to the base station are followed using Ant Colony Optimization to send the data.

SCHS: Smart Cluster Head Selection Scheme for Clustering Algorithms in Wireless Sensor Networks [5] states that intra-cluster communication is the main driving factor in energy efficiency of clustering protocol. The paper mentions that the energy consumed due to intra cluster communication depends on the position of the cluster heads within the cluster. Thus, this scheme has divided the network area into two parts: Border area and Inner area. Only the inner area nodes are eligible for becoming cluster heads and this approach reduces the intra cluster communication distance thereby improving the network lifetime. However, the nodes residing in the border area, though having higher energy values, never get a chance to become a cluster head. This leads to uneven distribution of energy within a cluster. 
Energy Efficient Clustering and Routing in Mobile Wireless sensor Network [1] proposes a novel hybrid multipath routing algorithm with an energy efficient clustering technique. Energy aware selection mechanism and Maximal nodal surplus energy estimation technique increase the energy performance during routing. It is mentioned that the algorithm proposed by this paper scale well in dynamic and energy efficient mobile sensor network. Here the entire sensor network area is assumed to be circumscribed into a big square which in turn is further divided into different square zones, each square zone being considered as a cluster. The cluster head nodes are termed as fusion nodes selected with a probability based on high residual energy, better transmission range and least mobility. The fusion nodes are responsible for inter cluster communications and can directly forward the data packet if the destination node is within their sensing zone. Otherwise, they follow a multi-hop path of fusion nodes to reach the destination node selecting the path with maximum surplus energy from a set of available paths.

Efficient Multilevel Clustering for Large-scale Heterogeneous Wireless Sensor Networks [3] proposes a multilevel clustering protocol by considering sensor nodes of different types. The sensor nodes vary in energy content and this heterogeneity is considered as a key factor for increasing the network lifetime in this paper. The algorithm takes into account the MRP or Minimum Reachability Power of each node to determine the cluster head. Based on the probability of becoming a cluster head the cluster heads are selected. The process of clustering stops when there is two or less number of regular nodes in the neighbor set of a cluster head.

Energy Efficient Cluster Based Routing Protocol (EECBRP) for Wireless Sensor Network [8] proposes an event-driven clustering technique in which a node that first senses the event initiates the cluster formation. It also follows a weight value based data forwarding to the base station and offers congestion control using backpressure technique.

\section{Proposed Work}

The proposed scheme EACBRS assumes the nodes of the network to be static or slightly mobile, that means mobility of the nodes are negligible and is not considered in this work. The base station or sink is of high configuration and is located outside the monitoring region. It is also assumed that all sensor nodes are capable of data aggregation, computing their own residual energy and are also GPS enabled. It is considered that the network is heterogeneous in nature, i.e. the amount of energy may vary from node to node in the network.

EACBRS is divided into seven modules, namely level formation, required number of cluster head selection, cluster formation, new node entry, data sensing and aggregation, data transmission to the base station and congestion control.

\subsection{Module 1: Level Formation}

The first phase of EACBRS is a level formation. This phase is initiated by the base station in the set-up phase and is not repeated in the lifetime of the network. At the beginning, all the nodes in the network are provided with a level_value $=0$. The base station checks the 
level_value of the nodes at 2-hop distance from it, and if they have a level_value $=0$, it sets the new level_value of those nodes as 1 . The nodes with level_value $=1$ form the first level of the network. Those nodes in turn check the level_value of the nodes at 2-hop distance from them. If the newly checked nodes have a level_value=0, they set the new level_value of the $\mathrm{i}^{\text {th }}$ node as follows:

$$
\text { level_value }_{\mathrm{i}}=\text { checking node's level_value }+1
$$

This process continues till no nodes are left with a level_value $=0$. The pseudo code of level formation is given below:

Initialization

STEP 1: Begin

STEP 2: If first round then,

a) for $i=1$ to total number of nodes set level_value $=0$

b) Set level_value of base station $=0$

c) Set found_neighbor $=0$ and updated_node $=0$

d) Base station calls Level_Formation( ) function.

STEP 3: End

Function Level_Formation( )

STEP 1: Begin

STEP 2: Base station forwards request message to all the nodes at two-hop distance from it.

STEP 3: for $\mathrm{i}=1$ to number of nodes at two-hop distance

if level_value is 0 then,

$$
\text { level_value }{ }_{\mathrm{i}}=1+\text { level_value of base station }
$$

STEP 4: for $\mathrm{i}=1$ to number of nodes with current highest level_value

if node $\mathrm{i}$ has a neighbor at two-hop distance and $\mathrm{D}_{\mathrm{B}}$ of the neighbor $>=$ $\mathrm{D}_{\mathrm{B}}$ of node i then, $/ / D_{B}$ is distance from the base station

a) forward request message to the neighbors.

b) found_neighbor++ //counting the number of neighbor nodes to which a request is forwarded

STEP 5: for i=1 to found_neighbor

if level_value ${ }_{i}==0$ then, level_value $=1+$ level_value of the node whose request is received by node i Inew level formed 
else

updated_node++ //counting the number of nodes whose level value is already updated

STEP 6: if updated_node $==$ found_neighbor then, //implies all nodes to which request was forwarded was already updated before

else go to Step 4

a) Send the level_value to the base station as highest level_value.

b) Stop level formation and go to Step 7

STEP 7: End

\section{Figure 1. Pseudo code for level formation}

\subsection{Module 2: Cluster Head Selection}

The second phase is the phase of cluster head selection. In this phase, first, the number of cluster heads required in each level is determined by the base station. The number of required cluster heads in a level is directly proportional to the average distance between the nodes of that level. If the nodes are scattered through a large area, it will be difficult for a smaller number of cluster heads to sense them all. The required number of cluster heads is also directly proportional to the total number of living nodes. When there is more number of living nodes, more cluster heads are needed for better coverage and load balancing. However, the required number of cluster heads is inversely proportional to the average distance of the nodes from the base station. The cluster head nodes near the base station are the only connecting link with the base station. Thus, those nodes are always included in any route from a sender cluster head of any level. If the lowermost level of the network has lesser number of cluster heads, then the same nodes will be selected over and over again for communicating with the base station. Therefore, by selecting more cluster heads in the lower levels will save energy. More number of cluster heads will help in proper distribution of energy. The following equation gives the required number of cluster heads in a level.

$$
\mathrm{C}_{\text {req }}=\left\lceil\mathrm{k} *\left\{\left(\frac{\mathrm{D}_{\text {avg }}}{\mathrm{DB}_{\text {avg }}}\right) * \mathrm{~N}_{\text {living }}\right\}\right]
$$

where $\mathrm{C}_{\text {req }}$ is the required number of cluster heads in level $\mathrm{i}, \mathrm{k}$ is a constant that depends upon the density of living nodes in the level, $\mathrm{D}_{\text {avg }}$ is the average distance between the nodes of level $i, D_{\text {avg }}$ is the average distance of the nodes of $i^{\text {th }}$ level from the base station and $\mathrm{N}_{\text {living }}$ is the number of active nodes of that level.

After determination of the $\mathrm{C}_{\text {req }}$ value for each level, the selection of cluster head starts on the basis of a competition bid value calculated by every node of each level. The competition bid value (CV) is calculated as: 


$$
\mathrm{CV}_{\mathrm{i}}=\frac{\mathrm{E}_{\mathrm{Ri}}}{\mathrm{D}_{\mathrm{i}}}
$$

where,

$$
D_{i}=\sum_{j=1}^{n} \frac{d_{i j}}{n}
$$

where $d_{i j}$ is the sum of the distances of node $i$ from each of its neighbor nodes $j$ lying in the same level, $n$ is total number of neighbor nodes of that node in that layer. $E_{R i}$ is remaining energy of the $\mathrm{i}^{\text {th }}$ node.

In each level, $\mathrm{C}_{\text {req }}$ numbers of nodes with the highest competition bid values (CV) are chosen as cluster head nodes. However, no node even with high CV can be chosen as cluster head if it is too close to any already selected cluster head of that level. If there is a node with the highest value of CV but lying in the sensing radius of an already chosen cluster head, then the next node with second highest CV is selected. In case of a race between two nodes having same CV, the one with the smaller node ID is selected as cluster head.

\subsection{Module 3: Cluster Formation}

After the completion of cluster head selection phase, the phase of cluster formation starts. In this phase, the selected cluster head nodes send a join request to the nodes of the same level, within their sensing radius. The format of the request is: \{join_REQ||nodeid||level_value\}.

The non-cluster head nodes join the cluster head node from which they receive the request. If a non-cluster head node receives a request from more than one cluster head node, it accepts only the first request and discards the rest. The cluster formation phase is repeated only when new nodes enter the network. Otherwise, the role of the cluster head is rotated among the nodes of the cluster when the residual energy of the current cluster head goes beyond the average residual energy of the cluster.

\subsection{Module 4: New Node Entry}

When a new node enters the network region, first the level_value of that node is determined on the basis of the position of the node. The node after entering the network forwards a request message to the nodes within its sensing radius. The format of the message is: \{Level_value_REQ||Node-id\} where Level_value_REQ is the request for obtaining its own level_value. The nodes receiving the request in turn send back an acknowledgement of the format: \{own_level_value||Node-id\} where own_level_value is the level_value of the node sending the acknowledgement. The level_value of the node whose acknowledgement is first received by the new node is set as the level_value for the new node also.

The $\mathrm{C}_{\text {req }}$ number is then re-calculated for the level of the new node to determine the required number of cluster heads for that level presently. Based on the new $\mathrm{C}_{\text {req }}$ value a new set of cluster heads is selected for that level using the cluster head selection procedure. The newly selected cluster heads, then repeat the cluster formation process. 


\subsection{Module 5: Data Sensing and Aggregation}

The next phase of EACBRS comprises of data sensing and aggregation. This module is initiated only when an event takes place within the network region. After completion of the previous phases, comprising of level formation, cluster head selection and cluster formation, the nodes in the network periodically check for events within their sensing radius. If no event is detected, they remain idle. Otherwise, the nodes start sensing of data relevant to that event. Data sensing phase may not start at the same time for all the clusters in the network. Only nodes which sense some event within their sensing radii start this phase in their clusters. Nodes of other clusters may remain idle at that time. When each node of a cluster where this phase is initiated, gathers a certain amount of data, it is forwarded to the cluster head of that cluster. A node waits till a certain amount of data is collected before forwarding it to the cluster head. This is done, so that less communication overhead takes place. The cluster head nodes receive the sensed data from their member nodes. They then remove the redundant data and aggregate them.

\subsection{Module 6: Data Transmission to the Base Station}

The sending of the aggregated data to the base station from each cluster head node in an energy efficient way is dealt in the last phase of EACBRS. In the last phase, all the cluster head nodes except those in the highest level, calculate a weight value $\mathrm{W}_{\mathrm{i}_{1}}$ for themselves. The Weight value $\mathrm{W}_{\mathrm{i}_{1}}$ is calculated as-

$$
\mathrm{W}_{\mathrm{i}_{1}}=\mathrm{k}_{1} *\left(\frac{\mathrm{ER}_{\mathrm{i}} * \mathrm{~N}_{\mathrm{i}}}{\mathrm{DB}_{\mathrm{i}}{ }^{2} * \mathrm{CR}_{\mathrm{i}} * \operatorname{load}_{\mathrm{i}}}\right)
$$

where $\mathrm{k}_{1}$ is the constant of proportionality taken as $=1 ; \mathrm{ER}_{\mathrm{i}}$ is the residual energy of cluster head $\mathrm{i}, \mathrm{N}_{\mathrm{i}}$ is number of cluster heads adjacent to cluster head $\mathrm{i} ; \mathrm{DB}_{\mathrm{i}}$ is distance between cluster head $\mathrm{i}$ and the base station, $\mathrm{CR}_{\mathrm{i}}$ is the rate of energy consumption of cluster head $\mathrm{i}$ which is calculated as: $=\frac{\Delta E}{\Delta T}$; $\operatorname{load}_{\mathrm{i}}$ is the number of packets already sent to cluster head $\mathrm{i}$ by other cluster head nodes and then send the weight value $W_{i_{1}}$ to the cluster heads of the next higher level, they can sense.

The cluster heads of the highest level first calculate the weight values $\mathrm{W}_{\mathrm{i}_{2}}$ for cluster head of the next lower level, within its sensing range, based on the following equation:

$$
\mathrm{W}_{\mathrm{i}_{2}}=\left(\frac{\mathrm{W}_{\mathrm{i}_{1}}}{\mathrm{D}_{\mathrm{N}}^{2}}\right)
$$

where, $\mathrm{D}_{\mathrm{N}}$ is distance of the current cluster head from the cluster head $\mathrm{i}$ of the next lower level to which it can send data.

Each cluster head node of the highest level then forwards the aggregated data to the cluster head of the next level, which has the highest weight value $W_{i_{2}}$ as calculated by 
itself. The cluster heads of lower level on the other hand, re-aggregates the data after receiving them from the higher level cluster heads and then forwards them to the next lower level in the same way. This process continues till the lowermost level, wherefrom each cluster head directly forwards the aggregated data to the base station.

\subsection{Module 7: Congestion Control}

Each node in the network maintains a queue. When a node is elected as a cluster head, all the active member nodes of that cluster head send their reading to it. It is not possible for the cluster head to process the data sent from all the nodes at the same time. So, the data packets from each node wait in the queue of the cluster head before being processed. Cluster head selects the data packets from the queue in a First-In-First-Out (FIFO) manner to process them. The processing time $\left(\mathrm{p}_{\mathrm{i}}\right)$ of a cluster head can be calculated as:

$$
\mathrm{p}_{\mathrm{i}} \equiv \frac{\operatorname{arr}_{\mathrm{i}}}{\left(\mathrm{sp}_{\mathrm{i}} * \mathrm{n}_{\mathrm{adj}}\right)} \quad \text { seconds }
$$

where, $\operatorname{arr}_{\mathrm{i}}$ is the rate at which packets arrive at the cluster head node, $\mathrm{sp}_{\mathrm{i}}$ is the processing speed of the cluster head node and $n_{\text {adj }}$ is the number of active member nodes sending data packets to the cluster head.

When the queue of the cluster head becomes full the cluster head gets congested and any more packets sent to it will get dropped. To avoid the dropping of packets by the cluster head a congestion control mechanism is adopted by EACBRS. The network congestion is controlled by controlling the network flow using concepts of queuing theory. The downstream flow of each node $i$ to node $j$ is controlled using the following equation:

$$
\text { Flow }_{\mathrm{i} \rightarrow \mathrm{j}}=\min \left(\mathrm{N}, \mathrm{b}_{\mathrm{ij}}+\mathrm{n}_{\mathrm{i}}\right)
$$

where, $\mathrm{N}$ is the maximum number of packets allowed to enter the destination before the queue becomes full, $b_{i j}$ is the number of packets blocked in the previous transfer, $n_{i}$ is the number of new packets of node $i$. The time for forwarding of data packets to the cluster head is broken into slots and at a particular time slot only one member node can forward the data packets to the cluster head using the flow control equation.

Similarly, while sending of the aggregated data to the base station, a cluster head node of a lower level may get congested by the packets sent from different cluster heads of the higher levels. The same congestion control procedure is adopted to control the upstream flow of each cluster head of the higher level to a cluster head of lower level. This congestion control mechanism adopted by EACBRS, not only reduces the number of packets dropped by a receiver node, but also saves the energy needed to resend the packets.

\subsection{Energy Consumption in EACBRS}

The energy consumed by a sensor node in transmitting a message can be denoted as:

$$
\mathrm{E}_{\mathrm{T}}=\mathrm{E}_{\mathrm{A}}+\mathrm{E}_{\mathrm{T} 0}
$$

where $E_{A}$ is the energy consumed by the power amplifier (PA) of the sensor node. $E_{A}$ is a function of distance and is given as: 


$$
\mathrm{E}_{\mathrm{A}}=\left(\mathrm{k}_{0} * \mathrm{r}_{\mathrm{ij}}^{2} * \mathrm{~s} * \mathrm{t}\right)
$$

where $\mathrm{k}_{0}$ is a constant that depends upon factors like hardware implementations, DC bias condition, operating frequency, load characteristics and amplifier's output; $r_{i j}$ is the distance between the sender node $i$ and the receiver node $j$; $s$ is the size of the packet sent and $t$ is the propagation delay.

$\mathrm{E}_{\mathrm{T} 0}$ is a constant amount of energy expressed as:

$$
\mathrm{E}_{\mathrm{T} 0}=\mathrm{E}_{\mathrm{B}}+\mathrm{E}_{\mathrm{F}}
$$

where $E_{B}$ is the energy consumption in the baseband DSP circuit and $E_{F}$ is the energy consumption in the front end circuit for transmitting a signal.

The energy required by a sensor node for receiving a signal can be expressed as:

$$
\mathrm{E}_{\mathrm{R}}=\mathrm{E}_{\mathrm{B}}+\mathrm{E}_{\mathrm{F}}+\mathrm{E}_{\mathrm{L}}
$$

where $E_{B}$ is the energy consumption in the baseband DSP circuit; $E_{F}$ is the energy consumption in the front end circuit and $E_{\mathrm{L}}$ is the energy consumption of the Low Noise Amplifier (LNA) for receiving a signal. In all the energy equations, energy is expressed in Joules.

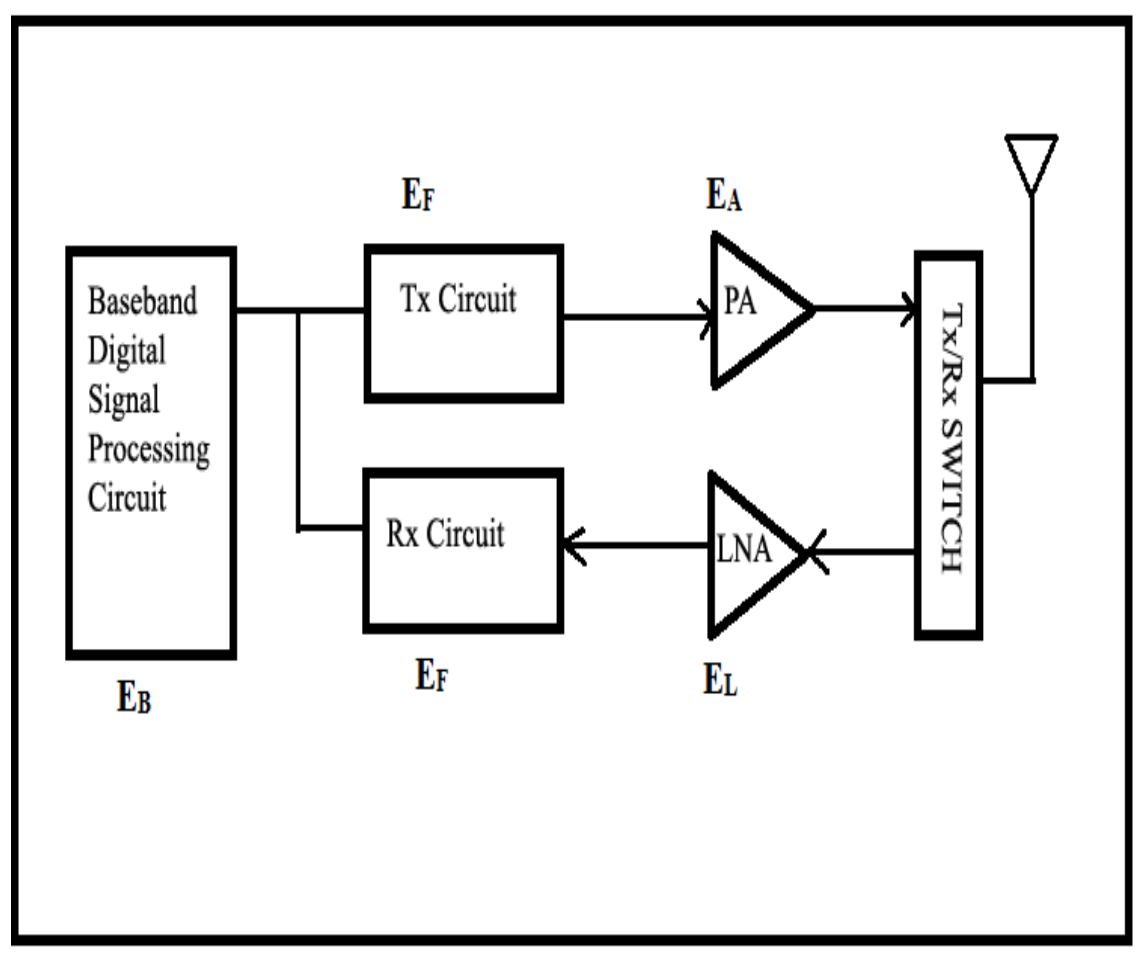

Figure 2. Communication module structure

A general flowchart of the proposed scheme is given in figure 3. 


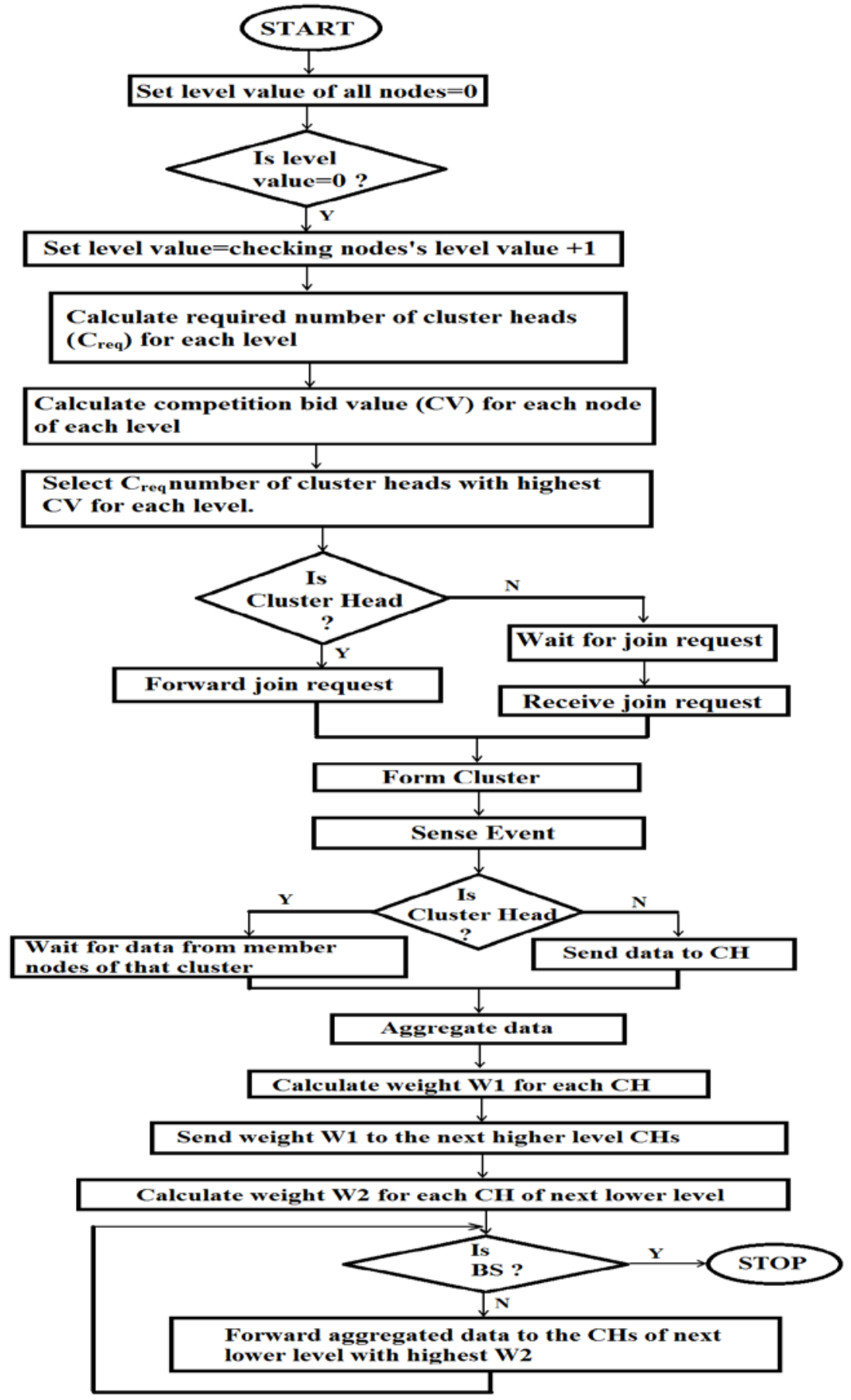

Figure 3. General flow chart of the routing scheme 


\section{Case Study}

To make the case study of EACBRS a network of 25 nodes is considered. Figure 4 shows the level formation of EACBRS. In the figure, the level_value is shown for each node. The base station first assigns the level_value for the nodes within 2-hop distance from itself as 1. Those nodes in turn assign the level_value for the nodes within 2-hop distance from them and so on.

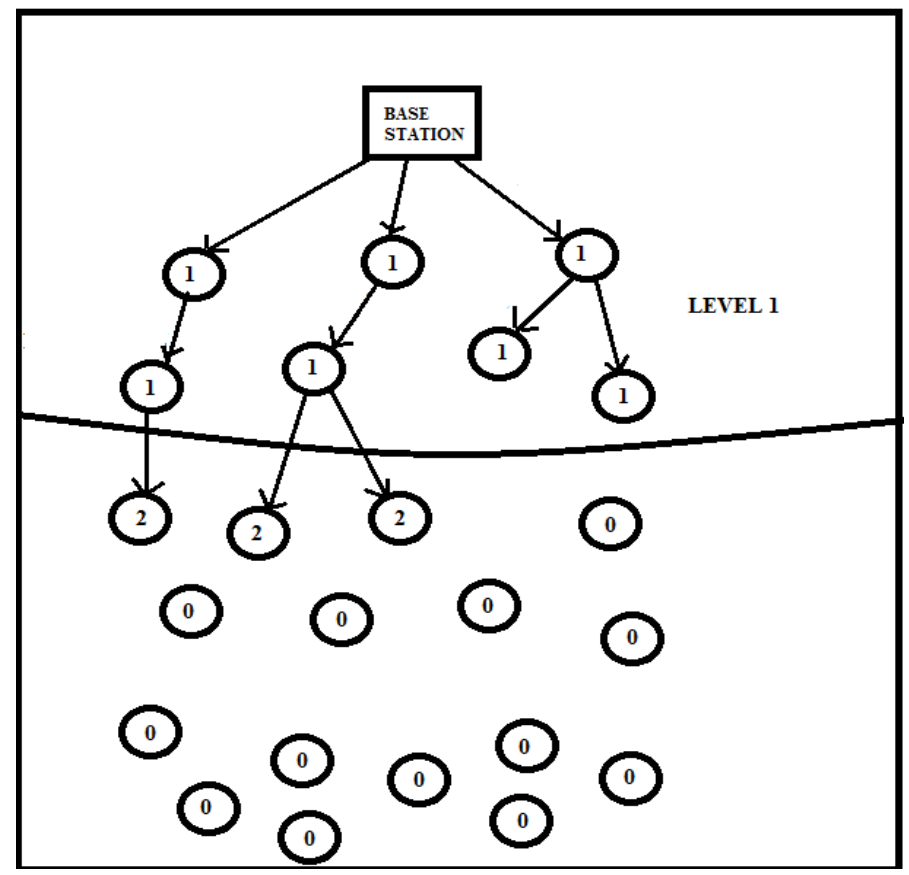

Figure 4. Level Formation

After level formation, the $\mathrm{C}_{\text {req }}$ of each level is determined. It is observed in Figure 5, that $\mathrm{C}_{\text {req }}$ of level 1 is 2 . Now each node of level 1 calculates their CV or competition bid value to become a cluster head. CV of all the nodes in level 1 is shown in Figure 5. First the node with the highest $\mathrm{CV}$, node 5 in this case, is selected as the first cluster head node of level 1. Then, it is found that the selected number of cluster heads is less than $\mathrm{C}_{\text {req. }}$ Thus, another cluster head is selected with second highest CV. It is not found that number of cluster heads selected in level 1 is equal to $\mathrm{C}_{\text {req. }}$. So, cluster head selection procedure for level 1 stops. In the similar manner, cluster heads of other levels are also selected. 


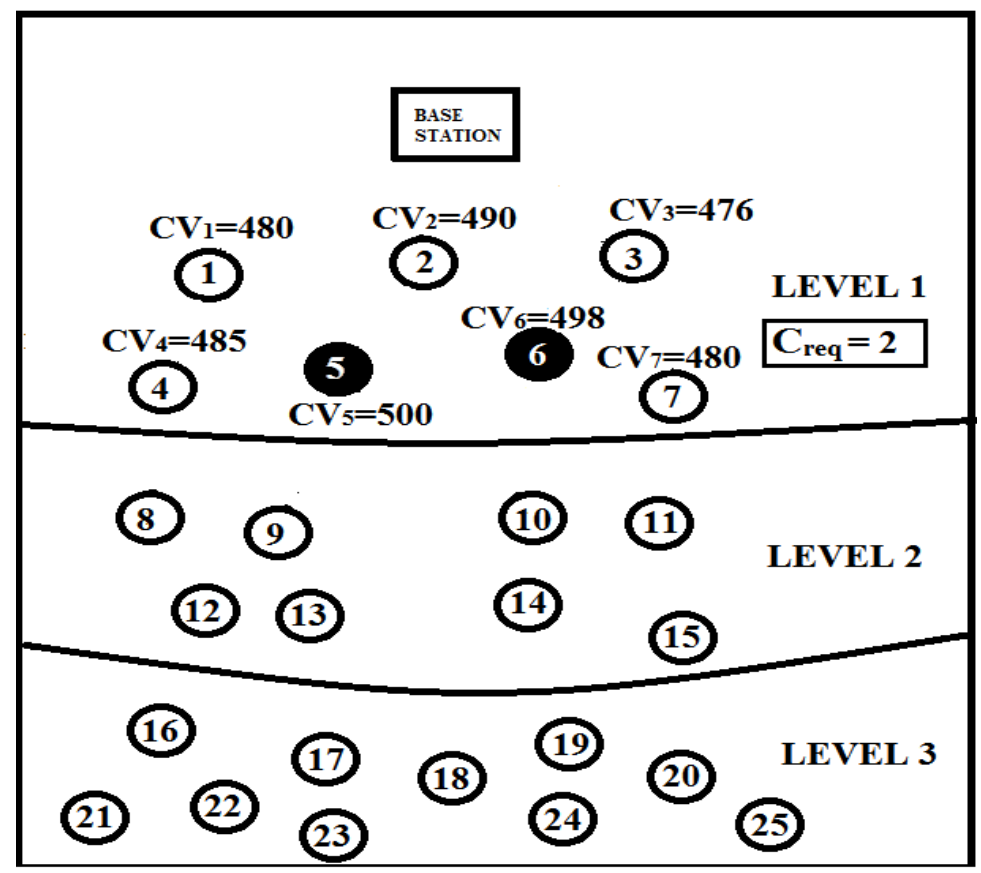

Figure 5. Cluster head selection

The selected cluster heads now forward a join request to the nodes within their sensing radii. Figure 6 shows this. In Figure 6, cluster head 5 sends join request to nodes 1, 2 and 4. On the other hand cluster head 6 sends join request to node 2, 3 and 7. Node 1 and 4 join cluster head 5; node 3 and 7 join cluster head 6 without any race. However, node 2 receives a join request from both the cluster head nodes. Since the join request from cluster head 5 came first to node 2, it joins cluster head 5 .

The data sensing phase is initiated only in the clusters where some nodes sense the occurrence of an event. The data sensing phase is illustrated in Figure 7. In Figure 7 an event takes place in level 2, near the clusters having cluster heads 13 and 14 . All the nodes in the two clusters sense the event and send the sensed data to the two cluster head of that cluster. The nodes residing in the other clusters remain idle at that point of time. The cluster heads after receiving the data aggregate them and compress them and makes them ready to be forwarded to the base station. 


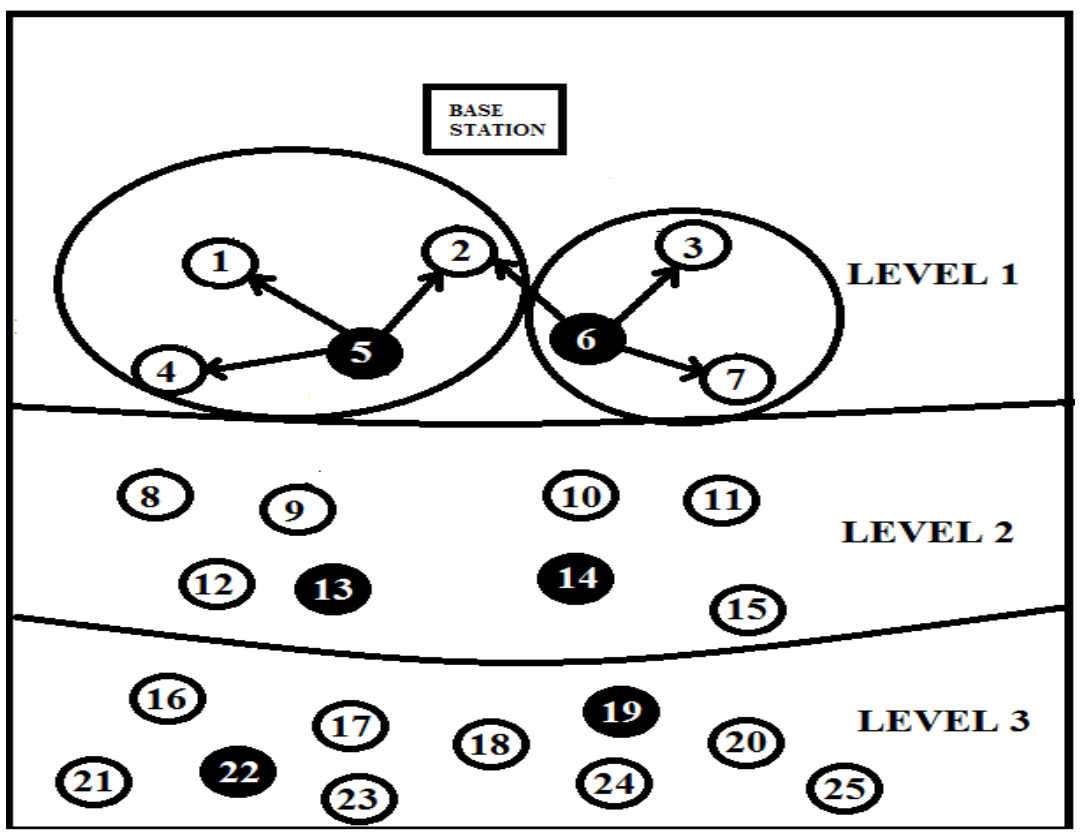

Figure 6. Cluster formation

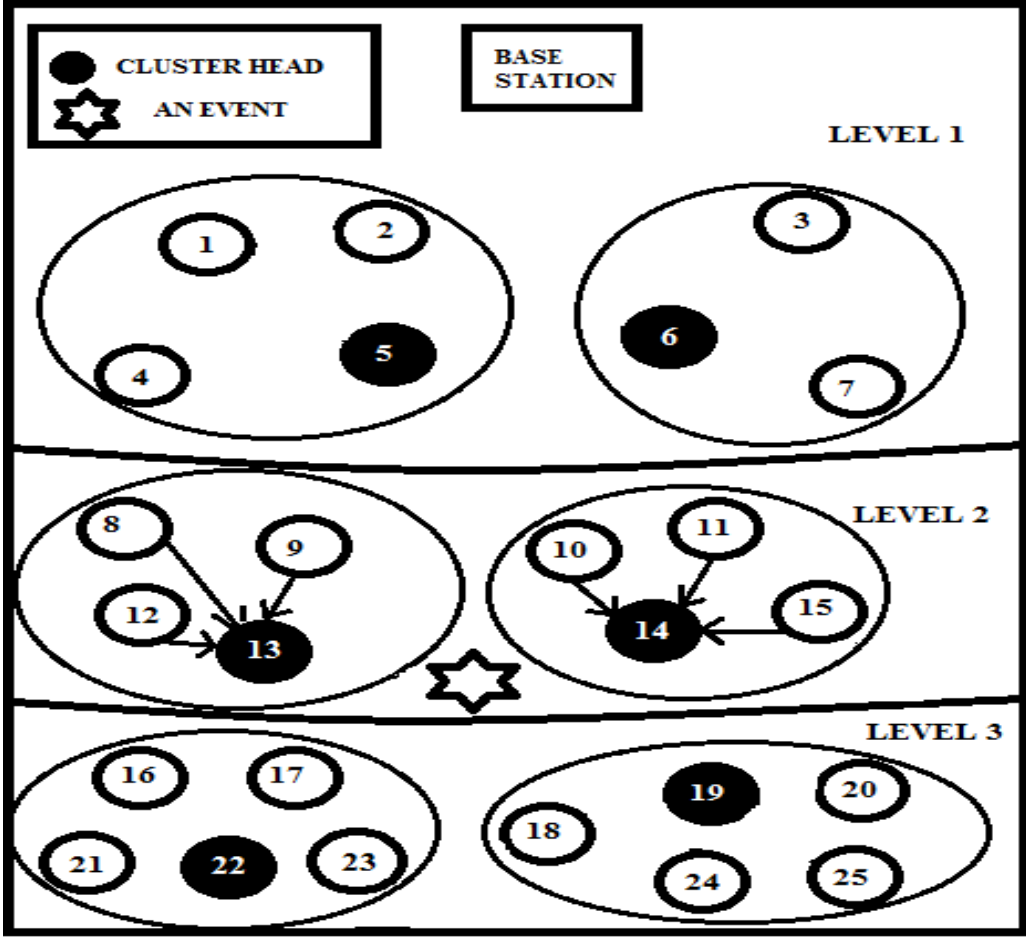

Figure 7. Data Sensing Phase 
In the last phase of EACBRS or sending of aggregated data to the base station phase, a weight value based data forwarding technique is adopted. In this phase, first the cluster heads of each level except the highest level, level 3 in this case, calculate their first weight value $\left(\mathrm{W}_{1}\right)$ and forward them to the cluster heads of next higher level. The cluster heads of higher level calculate the second weight value $\left(\mathrm{W}_{2}\right)$ for them. This phase is depicted in Figure 8. In the figure, $\mathrm{W}$ denotes $\mathrm{W}_{\mathrm{i} 2}$ calculated for node $\mathrm{i}$. In Figure 8, cluster head 19 can sense cluster head 13 and 14 of next lower level. The final weight value (W) calculated by 19 for node 13 and node 14 are 7.9 and 8.3 respectively. Thus, it forwards the aggregated data to node 14 of next lower level. Following this procedure all the other nodes forward their aggregated data towards the base station.

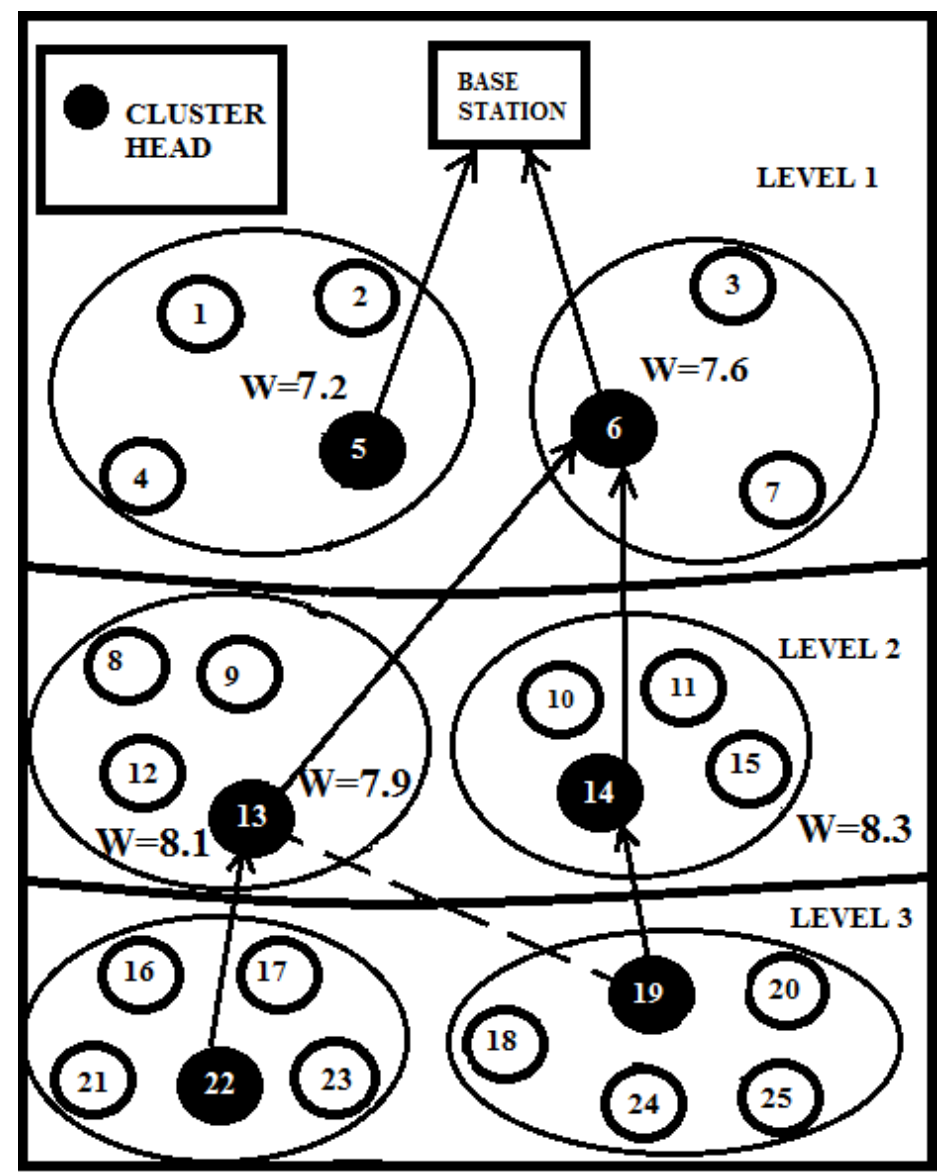

Figure 8. Data Sending to the Base Station 


\section{Simulation Results}

To analyze the performance of the algorithm, a network of 100 nodes is considered. It is assumed that the number of events taking place within 25 rounds is 25 . A round is defined as the total time taken in completing all the phases of an algorithm, once. The initial energy of each node is considered to be 50 Joules and the size of data packets are assumed to be of $10 \mathrm{~KB}$. The performance of EACBRS is observed by simulating the algorithm using NS2.29 and the results are compared with that of EEHC [4] and DEEC [6].

The parameter list is given in Table 1 .

Table 1. Parameter list

\begin{tabular}{|l|l|}
\hline Parameters & Description \\
\hline Number of nodes & 100 \\
\hline Network area & $100 \times 100 \mathrm{~m}^{2}$ \\
\hline Starting Energy & $50 \mathrm{~J}$ \\
\hline MAC Protocol & IEEE 802.15 .4 \\
\hline Number of rounds & 25 \\
\hline Sensor node & Imote2 \\
\hline Radio Frequency & $13 \mathrm{MHz}$ \\
\hline Time for each round & $10 \mathrm{~seconds}$ \\
\hline
\end{tabular}

\subsection{Average Residual Energy vs. Number of Rounds}

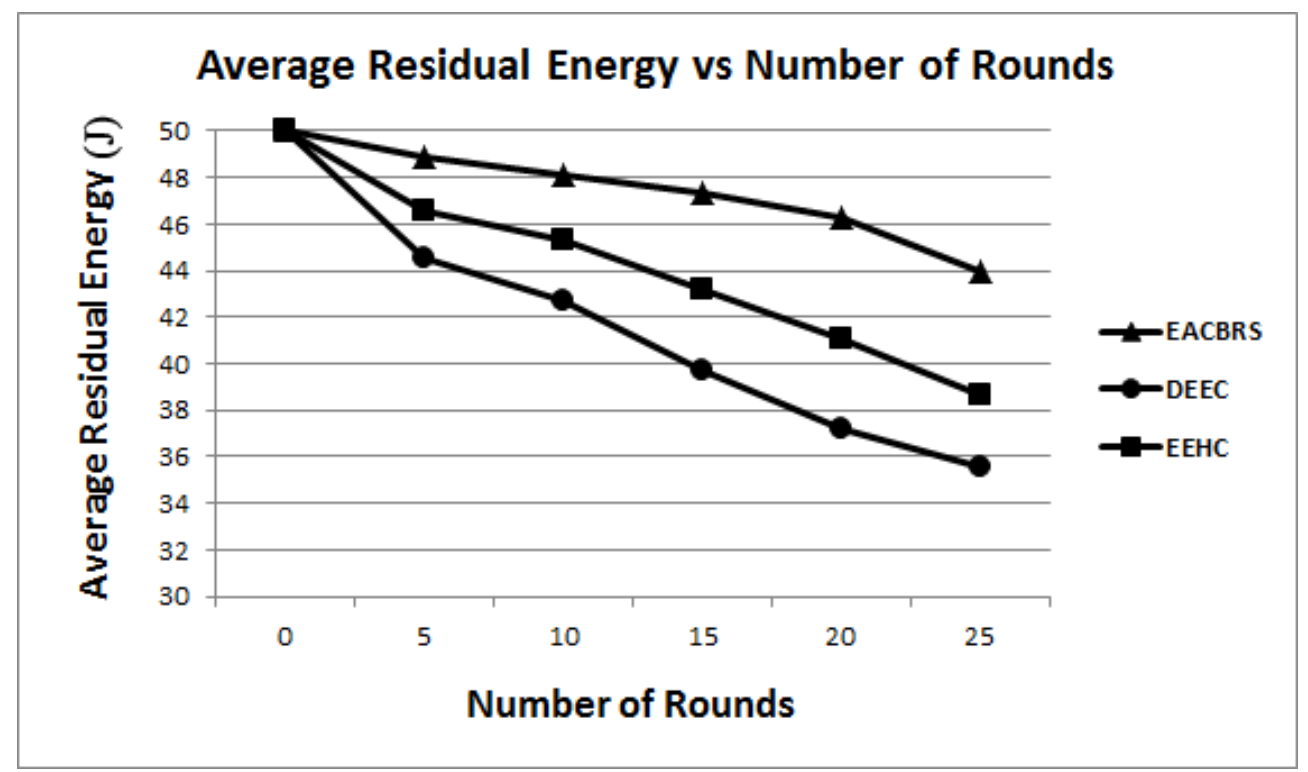

Figure 9. Average Residual Energy vs. Number of Rounds 
In Figure 9 the average residual energy of EACBRS is plotted against that of DEEC [6] and EEHC [4]. It is observed that even after 25 rounds the residual energy of EACBRS is more in the network as compared to the others. The selection of appropriate number of cluster heads for each level as well as avoiding the repetitive cluster formation for every round conserves much of the energy in case of EACBRS. The queuing theory based congestion control technique also prevents packet drop and saves the energy of packet re-transmission. Thus, it can be concluded that EACBRS conserves more energy and prolongs the lifetime of the network. Direct forwarding of data packets to the base station by EEHC [4] and DEEC [6] drains more energy.

\subsection{Number of Dead Nodes vs. Number of Rounds}

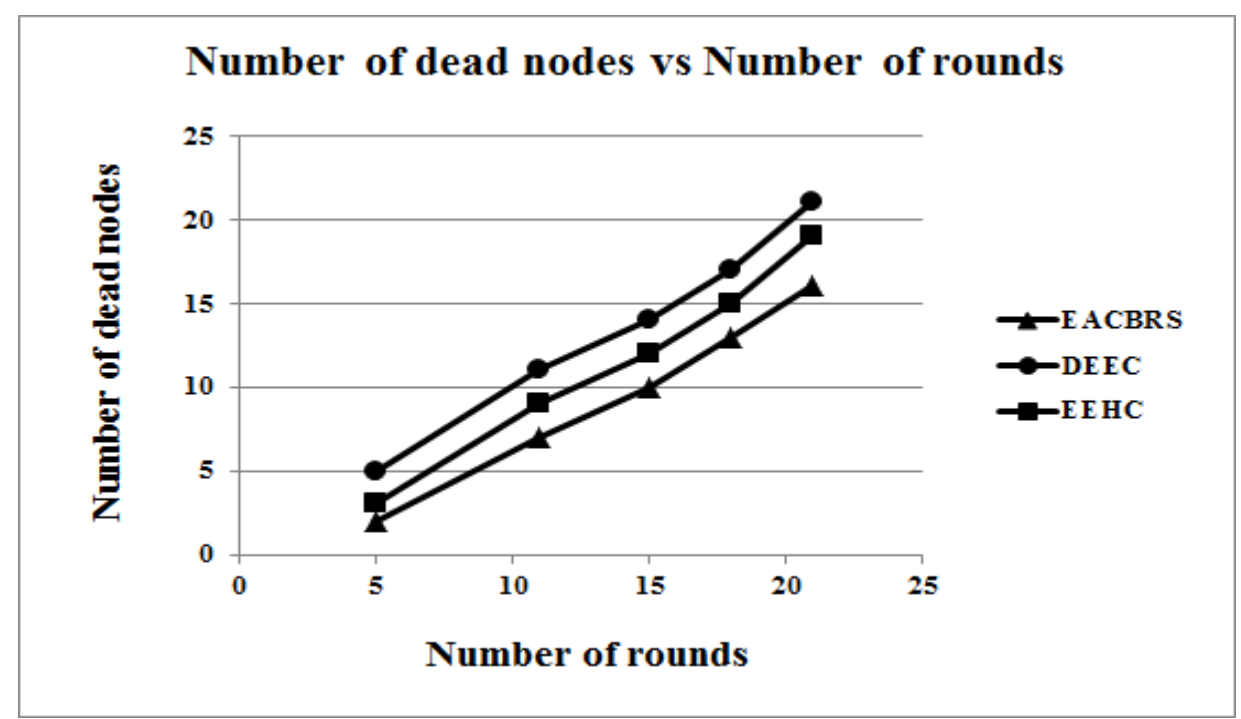

Figure 10. Number of Dead Nodes vs. Number of Rounds

In Figure 10 the number of dead nodes is plotted against the number of rounds for EACBRS, DEEC [6] and EEHC [4]. Dead nodes are the nodes that do not have a minimum amount of energy to send or receive a message. More dead nodes in a network imply less remaining lifetime of the network. It is noticed that more nodes are dead for EEHC and DEEC in comparison with EACBRS, after 25 rounds. So, EACBRS can prolong the network lifetime.

\subsection{Throughput vs. Load}

Figure 11 shows the throughput versus load graph. Load can be defined as the number of packets forwarded by different cluster heads and throughput is the number of packets successfully delivered to the base station. It is perceived that with the increase of load upto 50, the throughput increases for all the three algorithms. However, as the load increases 
beyond 50, the network gets congested in case of DEEC [6] and EEHC [4]. The network congestion results in packet loss and this leads to decreased throughput. In both DEEC and EEHC, there is also no congestion control mechanism to handle this situation. On the other hand, the flow control technique using queuing theory, adopted by EACBRS doesn't allow the nodes to forward packets more than the capacity of the receiver node's queue. This prevents the network from getting congested. Thus, the throughput of EACBRS becomes constant as the load increases beyond 50 .

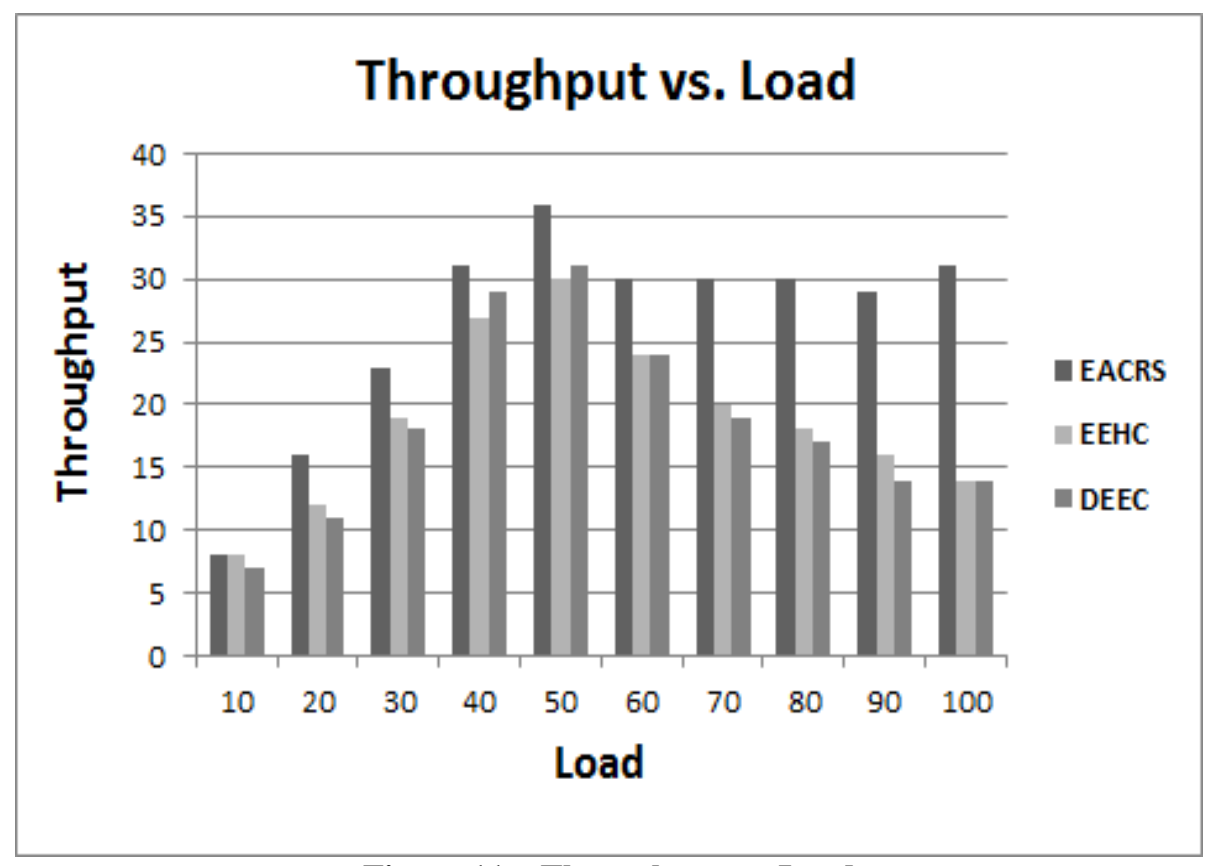

Figure 11. Throughput vs. Load

\subsection{Density vs. Delivery Rate}

Figure 12 shows the density versus delivery rate graph. Density is defined as the ratio of number of actual connections (AC) to the number of potential connections (PC) between nodes of a network. Potential connections can be calculated as-

$$
\mathrm{PC}=\frac{\mathrm{n} *(\mathrm{n}-1)}{2}
$$

Therefore, density is calculated as-

$$
\text { Density }=\frac{\mathrm{AC}}{\mathrm{PC}}
$$

Delivery rate is calculated as follows-

$$
\text { Delivery rate }=\frac{\text { Number of packets delivered to the base station }}{\text { transmission time }+ \text { propagation delay }}
$$


The propagation delay increases as the density of nodes increases since the hop-count for the packets increases, aggregation delay increases, cluster head selection delay increases, etc. The number of packets forwarded also increases with the increase in density. The increase in propagation delay becomes negligible compared to the number of packets sent. Thus the delivery rate increases with the increase in density for all the three algorithms. However, due to network congestion the delivery rate of EEHC and DEEC becomes less as compared to EACBRS.

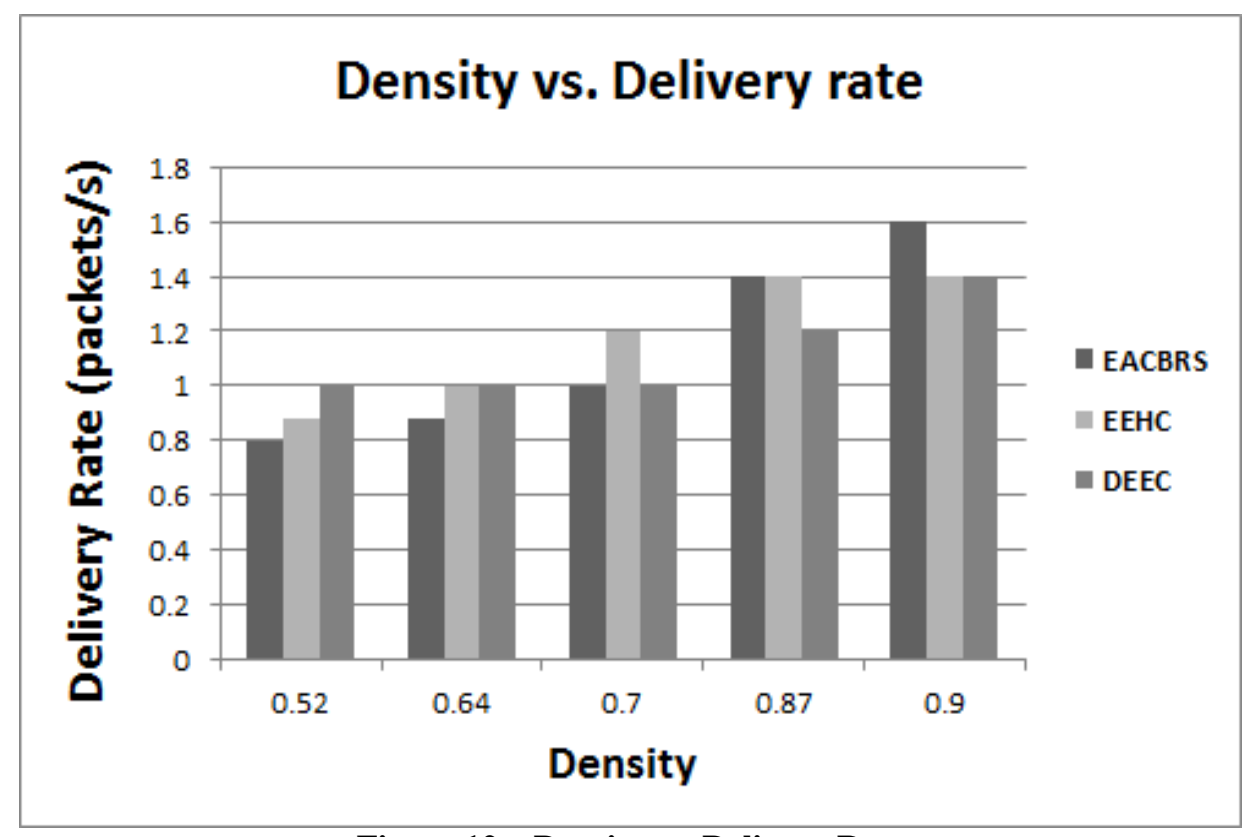

Figure 12. Density vs. Delivery Rate

\section{Conclusion}

The level based approach of EACBRS helps in area wise observation of the intent area. The base station plays the role of selecting the required number of cluster heads for each level and this result in proper distribution of the head nodes over the network region. The eventdriven data sensing avoids unnecessary data searching by the sensor nodes and this also conserves energy. Cluster formation is not repeated after each round and it is repeated only when new nodes enter the network region. Thus, the time taken for cluster formation in each round and the delay occurred in the data forwarding is avoided in EACBRS. The queuing theory based congestion control technique adopted by the proposed scheme not only increases the throughput by averting the nodes from getting congested but also preserves the energy of data retransmission. The simulation results illustrate how the proposed scheme EACBRS performs better than the existing routing algorithms like DEEC [6] and EEHC [4]. 


\section{References}

[1] Getsy S Sara, Kalaiarasi.R, Neelavathy Pari.S., Sridharan D., Energy Efficient Clustering and Routing in Mobile Wireless sensor Network, International Journal of Wireless \& Mobile Networks (IJWMN), Vol.2(4), pp. 106-114, November 2010.

[2] Heinzelman W.B., An application-specific protocol architecture for wireless microsensor networks, IEEE Transactions on Wireless Communications, Vol. 1 (4), pp. 660-670, 2002.

[3] Katiyar Vivek, Chand Narottam, Soni Surender, Efficient Multilevel Clustering for Large-scale Heterogeneous Wireless Sensor Networks, in Proceedings of 2011 International Conference on Communication, Computing \& Security, ICCCS '11, pp. 1-6, doi:10.1145/1947940.1947942.

[4] Kumar Dilip, Patel R.B., Aseri Trilok C., Energy Efficient Heterogeneous Clustered scheme for Wireless Sensor Network, Journal of Computer Communications, Elsevier, Vol. 32 (4), pp. 662-667, March 2009.

[5] Pal Vipin, Singh Girdhari, Yadav Rajender Prasad, SCHS: Smart Cluster Head Selection Scheme for Clustering Algorithms in Wireless Sensor Networks, Wireless Sensor Network, Vol. 4, pp. 273-280, 2012.

[6] Qing Li, Zhu Qingxin, Wang Mingwen, Design of a distributed energy-efficient clustering algorithm for heterogeneous wireless sensor networks, Journal of Computer Communications, Elsevier, Vol. 29, pp. 2230-2237, 2006.

[7] Roy Sohini and Das Ayan Kumar, Cluster based Event Driven Routing Protocol (CERP) for Wireless Sensor Network, International Journal of Computer Applications (IJCA), Vol.88 (11), pp.6-11, February 2014.

[8] Roy Sohini and Das Ayan Kumar, Energy Efficient Cluster Based Routing Protocol (EECBRP) for Wireless Sensor Network, in Proceedings of First International Conference on Networks \& Soft Computing (ICNSC'14), published by IEEE, pp. 3640, 2014.

[9] Sharma Ajay K., Kour Harneet, Hybrid Energy Efficient Distributed Protocol for Heterogeneous Wireless Sensor Network, Internatioanl Journal of Computer Applications (IJCA), Vol.4 (6), pp. 660-669, July 2010.

[10] Yang Jing, Xu Mai, Zhao Wei, Xu Bhaoguo, A Multipath Routing Protocol Based on Clustering and Ant Colony Optimization for Wireless Sensor Networks, Sensors 2010, Vol. 10(5), pp.4521-4540; doi: 10.3390/s1005040521.

Received 13.10.2014, accepted 28.05.2015 